Fatty Aldehyde Dehydrogenase

\title{
(ALDH3A2)-Dependent Neutralization of Advanced Lipid Peroxidation End Products (ALEs) at the Bifurcation of Hormetic and Degenerative Pathways in Pancreatic Beta Cells ${ }^{+}$
}

\author{
Shlomo Sasson \\ Institute for Drug Research, Faculty of Medicine, The Hebrew University, Jerusalem 9112001, Israel; \\ shlomo.sasson@mail.huji.ac.il \\ † Presented at Natural Products and the Hallmarks of Chronic Diseases-COST Action 16112, Luxemburg, \\ 25-27 March 2019.
}

Published: 16 April 2019

\begin{abstract}
Hyperglycemia and hyperlipidemia synergistically and adversely impair insulin secretion and ultimately lead to pancreatic beta cell decomposition. We found that both nutrient overload conditions displace arachidonic and linoleic acids from membrane phospholipids and subject them to free radical-mediated peroxidation and generation of advanced lipid peroxidation end products (ALEs), of which the aldehyde 4-hydroxy-2-nonenal (4-HNE) is prominent. When present at high levels this electrophilic molecule binds covalently to nucleophilic moieties in proteins, phospholipids and nucleic acid, modifies their structure and function and leads to severe cellular dysfunction and apoptosis. However, when present at low and unharmful levels this same molecule activates the nuclear receptor PPARS and augments insulin secretion. The level of endogenous 4-HNE is determined by the extent of lipid peroxidation on one hand, and by enzymatic neutralization of the aldehyde, on the other. The latter step is mediated by enzymatic processes of which the transformation of the aldehyde to the corresponding inactive carboxylic derivative 4-hydroxy-2-nonenoic acid (4-HNA) is significant. The enzyme responsible for this transformation, which belongs to the large family of aldehyde dehydrogenases and selectively neutralizes fatty acid-derived aldehydes, is ALDH3A2, which is also known as fatty aldehyde dehydrogenase (FALDH). Consequently, we hypothesized that the expression level and function of ALDH3A2 may determine the fate of beta cells under nutrient overload conditions: insufficient neutralization of 4-HNE by the enzyme will lead to cell demise, whereas increased expression and function will extend the adaptive response of beta cells. This adaptive response that is characterized with increased insulin secretion enables effective storage of the nutrient surplus in peripheral tissues and organs while minimizing the dire consequences of the nutrient overload. We aimed at investigating the expression pattern of ALDH3A2 in pancreatic beta cells (the INS-1E cell line) under hyperglycemic condition without or with supplementation with saturated fatty acids (e.g., palmitic acid). Our results show significant glucose- and palmitic acid-dependent induction of ALDH3A2 expression in the cells. We also found that the transformation of palmitic acid (16:1) to mono-unsaturated palmitoleic acid $(16: 1$, cis 9$)$ by the enzyme Stearoyl-CoA desaturase-1 (SCD1) decreased the burden of the lipid stress on the cells and abrogated the stimulus for the induction of ALDH3A2. Preliminary experiments indicated that the upregulation of the induction of ALDH3A2 was partly induced by PPAR $\delta$. These findings correlate to our previous discovery that the hormetic effects of 4-HNE were mediated via activation of this nuclear receptor. In summary, this study assigns a central role to the enzyme ALDH3A2 in the protective mechanism beta cells employ to
\end{abstract}


mitigate detrimental effects of ALEs, and divert them into hormetic agents, that by feedback mechanism through PPARס increase ALDH3A2 expression.

Keywords: aldehyde dehydrogenase; ALDH3A2; beta cells; 2; type 2 diabetes; lipid peroxidation; 4-hydroxynonenal

Funding: This research was funded by grants from the Brettler Center for Research of Molecular Pharmacology and Therapeutics and the David R. Bloom Center for Pharmacy at the Hebrew University of Jerusalem (2016, 2018).

Acknowledgments: The author wishes to acknowledge the dedicated work of Elisheva Gur in the project. This work is based upon work from COST Action NutRedOx-CA16112 supported by COST (European Cooperation in Science and Technology).

Conflicts of Interest: The author declares no conflict of interest.

(c) 2019 by the authors. Licensee MDPI, Basel, Switzerland. This article is an open access article distributed under the terms and conditions of the Creative Commons Attribution (CC BY) license (http://creativecommons.org/licenses/by/4.0/). 